\title{
INCISIONES LIMBARES RELAJANTES Y CIRUGÍA DE LA CATARATA: NUESTRA EXPERIENCIA
}

\section{LIMBAL RELAXING INCISIONS AND CATARACT SURGERY: OUR EXPERIENCE}

\author{
COLOMA-GONZÁLEZ Iㅁ, GONZÁLEZ-HERRERA Mํ, MENGUAL-VERDÚ E², \\ HUESO-ABANCENS JR ${ }^{2}$
}

\section{RESUMEN}

Objetivo: Evaluar la reducción del astigmatismo corneal preexistente en la cirugía de catarata mediante incisiones limbares relajantes (ILRs).

Método: Estudio prospectivo de dos grupos de pacientes (tratamiento y control) con astigmatismo prequirúrgico $\geq 1 \mathrm{D}$. Los 30 pacientes del grupo control fueron sometidos a facoemulsificación por incisión temporal en córnea clara y los 32 pacientes del grupo tratamiento a ILRs junto a facoemulsificación por incisión temporal en córnea clara. Se usó el test de Holladay para valorar la eficacia del tratamiento. Asimismo, presentamos un pequeño estudio de la prevalencia y distribución del astigmatismo en nuestra población de trabajo.

Resultados: El seguimiento promedio de los 62 pacientes fue de 3 meses, con un cambio astigmático medio a los 3 meses de $-0.55 \mathrm{D}(-0,75$ a $-0,35)$ en el grupo tratamiento y de $0.04 \mathrm{D}(-0,3$ a 0,3$)$ en el grupo control $(\mathrm{p}<, 000)$.

\begin{abstract}
Purpose: To evaluate the reduction of pre-existing corneal astigmatism at the time of cataract surgery with limbal relaxing incisions (LRIs).

Methods: A prospective study of two groups of patients (treatment and control) with pre-existing astigmatism $\geq 1 \mathrm{D}$ was performed. The 30 patients in the control group had a temporal clear corneal phacoemulsification and the 32 patients of treatment group had combined temporal clear corneal phacoemulsification and LRIs. Holladay analysis was used to assess the efficacy of treatment. An astigmatism distribution and prevalence study in our population is also presented.

Results: Three months after surgery, the mean astigmatism change was $-0.55 \mathrm{D}(-0.75$ to -0.35$)$ in the treatment group and $0.04 \mathrm{D}(-0.3$ to 0.3$)$ in the control group $(\mathrm{p}<.0001)$.
\end{abstract}

Conclusions: LRI is a simple, safe and effective method not only for reducing pre-existing astigma-

\footnotetext{
Recibido: 31/5/06. Aceptado: 17/7/07.

Hospital Universitario San Juan de Alicante. San Juan de Alicante. España.

1 Licenciado en Medicina.

2 Doctor en Medicina.

Correspondencia:

Itziar Coloma González

Hospital Universitario San Juan de Alicante

Ctra. Nacional Alicante-Valencia, N-332, s/n

03550 San Juan (Alicante)

España

E-mail: icg_ct@yahoo.es
} 
Conclusiones: Las ILRs son un método sencillo y efectivo para conseguir reducir el astigmatismo previo durante la cirugía de catarata y con ello la dependencia de gafa.

Palabras clave: Incisiones limbares relajantes, facoemulsificación, cirugía de catarata, astigmatismo, emetropía. tism during cataract surgery but in providing good unaided visual acuity (Arch Soc Esp Oftalmol 2007; 82: 551-554).

Key words: Limbal relaxing incisions, phacoemulsification, cataract surgery, astigmatism, emmetropia.

\section{INTRODUCCIÓN}

En los últimos años nuestro fin con la cirugía de la catarata ha pasado de ser la simple extracción a conseguir la mejor agudeza visual (AV) sin corrección y, con ello, reducir la dependencia de gafa, tanto de cerca como de lejos. Por eso se puede considerar hoy día ya como «cirugía refractiva de la catarata».

Este cambio ha sido posible gracias a la integración de los avances técnicos y teóricos, movido todo ello por el grado de exigencia cada vez mayor por parte del oftalmólogo y del paciente.

Esto implica que, a la hora de planificar la cirugía, debemos de tener en cuenta tanto el componente esférico como el astigmático si se quiere conseguir nuestro objetivo que es la emetropía. El componente esférico lo compensaremos mediante la lente intraocular (LIO) implantada, por ello, se analizarán todos los factores a la hora de elegir el tipo de lente y su potencia en función de los datos biométricos obtenidos con un buen aparato y mejor técnico, fórmula y constante apropiadas, así como, las características del paciente y la técnica quirúrgica que tengamos pensado realizar. El componente astigmático se planificará en función de la queratometría y/o topografía corneal principalmente pero también se han de tener en cuenta otros factores como la edad, el ojo contralateral, las características corneales... Eligiendo de entre las opciones técnicas la más apropiada para nuestro paciente.

\section{SUJETOS, MATERIAL Y MÉTODOS}

Se incluyeron en nuestro estudio 62 pacientes cataratosos con $\geq 1 \mathrm{D}$ de astigmatismo programados para implante de LIO por un mismo cirujano y se dividió en dos grupos (control y tratamiento) de manera aleatoria. En el grupo tratamiento los pacientes fueron sometidos a facoemulsificación por incisión temporal en córnea clara, implante de
LIO multifocal y realización de ILRs según nomograma de Dr. Louis D. Nichamin (fig. 1). A los pacientes del grupo control se les realizó facoemulsificación por incisión temporal en córnea clara más implante de LIO monofocal.

Se excluyeron pacientes con patología corneal, astigmatismo irregular, portadores de lentes de contacto y pterigión.

Se realizó a todos ellos un examen oftalmológico completo que incluyó agudeza visual (AV) sin corrección y con ella, queratometría y autorrefractometría, tonometría, paquimetría y contaje endotelial, biomicroscopía y fundoscopía.

Para realizar las ILRs, previo a la cirugía con el paciente sentado en la lámpara de hendidura, se realizó el marcaje del meridiano horizontal $\left(0^{\circ}-180^{\circ}\right)$ con rotulador violeta de genciana. De esta manera no se pierde su orientación tras la rotación ocular que tiene
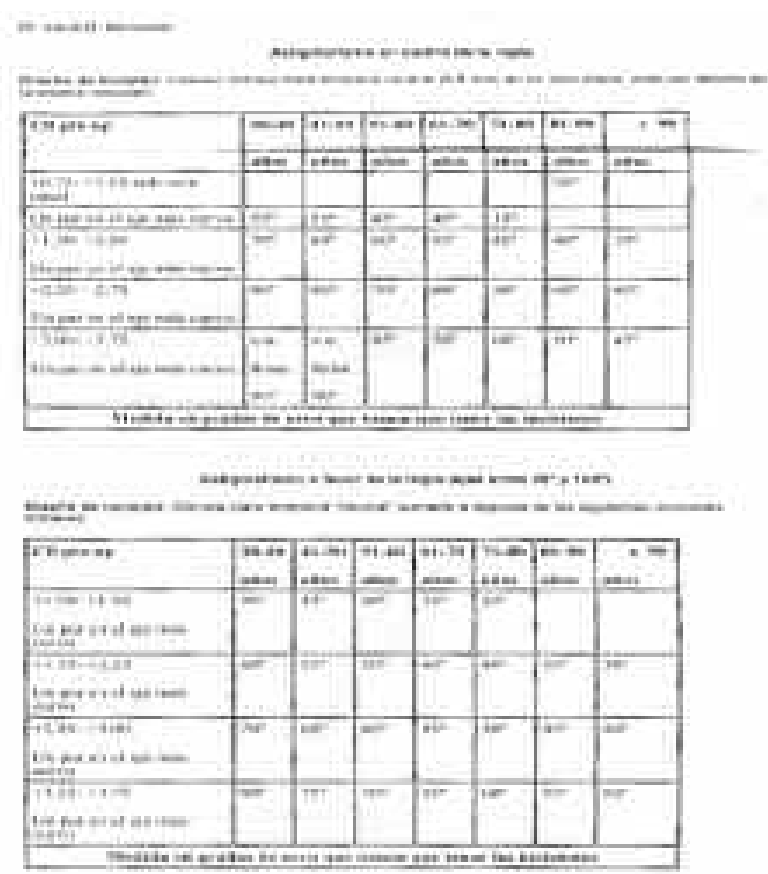

Fig. 1: Nomograma del Dr. Louis D. Nichamin. 
lugar al posicionar al paciente en la camilla. Una vez dentro de quirófano se procede al marcaje de las ILRs a realizar mediante el anillo de Méndez y un cuchillete de $15^{\circ}$. Y, finalmente, se realizan 1 ó 2 incisiones arqueadas en córnea clara perilímbica con cuchillete de diamante precalibrado a $600 \mu(500 \mu$ si $\geq 80$ años).

La facoemulsificación e implante de LIO se realizó a través de una incisión de $2.20 \mathrm{~mm}$ en córnea clara temporal $\left(\approx 30^{\circ}\right.$ en los ojos derechos y $30^{\circ}$ en los izquierdos) y se aseguró la estanquidad de la misma mediante punto corneal suelto con nylon 10/0, que fue retirado al mes de la intervención.

A los pacientes con ILRs se les colocó una lentilla terapéutica tras el lavado de las mismas con BSS que fue retirada al día siguiente de la intervención.

Todos los pacientes recibieron pauta de antibiótico y corticoesteroide durante un período mínimo de 6-8 semanas tras cirugía.

Se midió la queratometría a las 6 semanas y a los 3 meses, descartando para el análisis estadístico los valores recogidos a las 6 semanas por la relativa proximidad temporal con la retirada de la sutura corneal. Los datos fueron analizados mediante el test de $\mathrm{T}$ de student para datos independientes con el fin de valorar posibles diferencias entre ambos grupos. Se realizó un análisis estadístico con el paquete informático SPSS versión 10 (SPSS for Windows, SPSS Inc, Chicago, USA).

\section{RESULTADOS}

La aleatorización de la muestra evita posibles sesgos de selección y hace comparables ambos grupos de estudio incluso en los valores queratométricos preoperatorios (control 1.51, 0.5 DE; tratamiento $1.53,0.47 \mathrm{DE})$.

Para el análisis del cambio astigmático ocurrido tras la cirugía se utilizó el test de análisis vectorial de Holladay por ser el que más información aporta sobre el efecto que tiene el propio procedimiento quirúrgico en sí al describir la relación entre el cilindro inicial y el resultante (1). En el grupo control encontramos una reducción media del astigmatismo prequirúrgico de $-0,55 \mathrm{D}$, rango $-0,75$ a $-0,35 \mathrm{D}$, a los 3 meses de la cirugía. En el grupo control el cambio medio fue de $0,04 \mathrm{D}$; rango $-0,3$ a 0,3 D. Y, al comparar los resultados (medias de la diferencia) de ambos grupos mediante análisis con la $\mathrm{T}$ de student para datos independientes, encontramos una diferencia clínicamente significativa $(\mathrm{p}<, 000)$. Por lo tanto, las ILRs ejercen un mayor cambio astigmático y de manera más controlada que la incisión tradicional para facoemulsificación.

Expresando de manera gráfica los resultados de ambos grupos según el cambio astigmático (cilindro final-cilindro inicial) se aprecia que en el grupo control la tendencia central es a 0 (no cambio) mientras que en el grupo tratamiento es a la reducción del astigmatismo preoperatorio (valores negativos) (fig. 2).

No se produjo ningún caso de astigmatismo irregular y sólo uno de hipercorrección <1D. Las molestias referidas por los pacientes fueron similares en ambos grupos (picor, lagrimeo, sensación de cuerpo extraño...).

\section{DISCUSIÓN}

Los avances en la facoemulsificación y las LIOs han permitido conseguir AV corregidas tras la cirugía $\geq 0,5$ en aproximadamente el $90 \%$ de los ojos en general, y del $95 \%$ de los ojos sin enfermedad ocular previa (2). Sin embargo, con ella siempre se consigue un mejor control del componente esférico que del astigmático y si lo que pretendemos es conseguir un resultado postquirúrgico lo más próximo a la emetropia se vuelve imprescindible también su correción, o al menos reducción. Existen diferentes opciones para conseguir reducir el astigmatismo preexistente durante la cirugía de catarata. Entre ellas encontramos técnicas incisionales, anillos intracorneales, LIOs tóricas y cirugía de láser excimer.

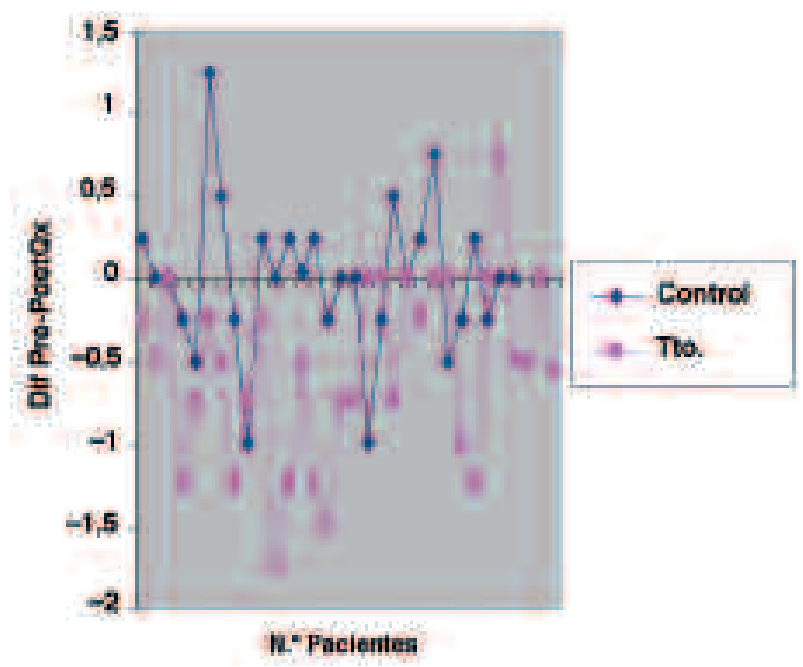

Fig. 2: Representación gráfica de los resultados obtenidos en ambos grupos tras cirugía: Cambio astigmático. 
Teniendo en cuenta que, según un estudio realizado en nuestro servicio, aproximadamente el $63 \%$ de nuestros pacientes con cataratas tienen un astigmatismo $\geq 1 \mathrm{D}$ y un $30 \%$ entre $1-1.5 \mathrm{D}$ (fig. 3), optamos por incluir en nuestro protocolo quirúrgico la realización de alguna técnica incisional corneal, en concreto las ILRs. Ésta técnica consiste en la realización de unos pequeños cortes curvilíneos a nivel del limbo que actúan cambiando la curvatura corneal. Producen un aplanamiento del meridiano sobre el que se realizan por efecto de la adición de tejido (3) y a la vez incurvan el meridiano perpendicular (efecto de acoplamiento) $(1,4)$.

Nuestros resultados demuestran su efectividad a la hora de reducir el astigmatismo prequirúrgico $\mathrm{y}$, además, nos resultan especialmente útiles en nuestro medio puesto que habitualmente nos encontramos ante un astigmatismo moderado, el cual controlan muy bien. Asimismo, la curva de aprendizaje y el material necesario (fig. 4) son mínimos, al igual que las complicaciones observadas.

Consideramos más apropiado realizar las ILRs previo a la facoemulsificación por los cambios en el espesor corneal que tienen lugar durante la misma que podrían alterar los resultados. Y atribuímos el menor efecto de esta técnica observado en nuestro estudio comparado con el observado en otros de características similares (5) al empleo de sutura para la incisión principal, aunque precisaríamos de

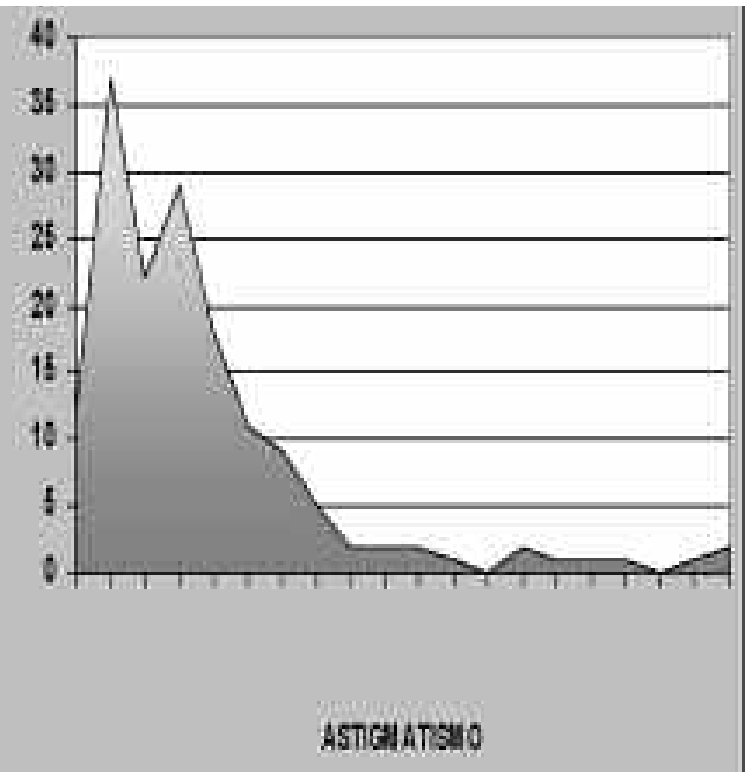

Fig. 3: Representación gráfica de la distribución del astigmatismo en nuestra población.

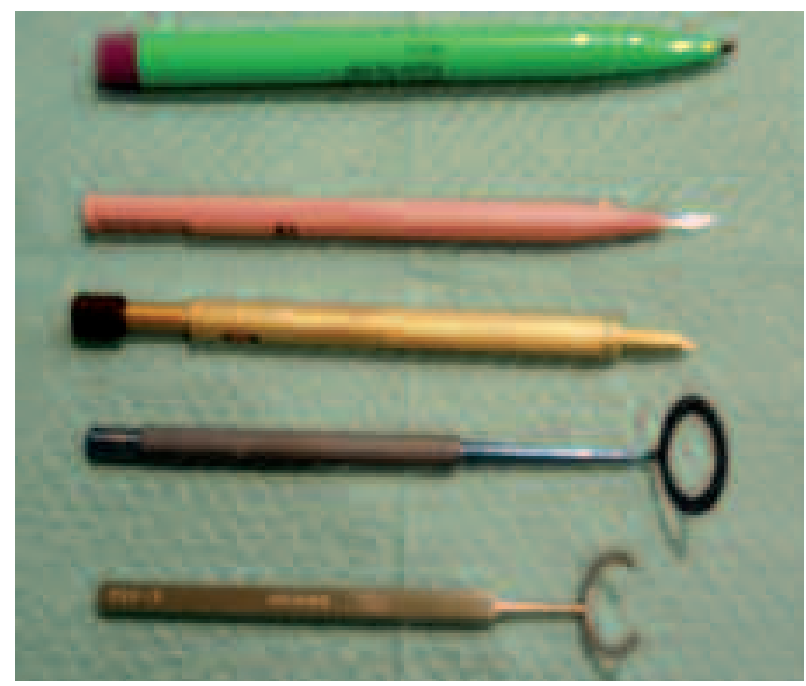

Fig. 4: Material quirúrgico empleado para las incisiones limbares relajantes.

un nuevo estudio sin su uso para estar seguros de que sea esa la única causa.

Entre las ventajas que observamos al asociar una técnica de control del astigmatismo a la facoemulsificación e implante de LIO multifocal damos especial importancia a la reducción de la necesidad de corrección óptica para la vida diaria y la mejor visión estereoscópica.

Como desventajas apreciables mencionar el posible debilitamiento corneal que pueden suponer para estas córneas ya seniles y la regresión de su efecto con el tiempo.

En conclusión, consideramos que las ILRs son sencillas, seguras y efectivas a la hora de reducir el astigmatismo corneal prequirúrgico durante la cirugía de catarata.

\section{BIBLIOGRAFÍA}

1. Morlet N, Minassian D, Dart J. Astigmatism and the analysis of its surgical correction. Br J Ophthalmol 2001; 85: 1127-1138.

2. Westcott MC, Tuft SJ, Minassian DC. Effect of age on visual outcome following cataract extraction. Br J Ophthalmol 2000; 84: 1380-1382.

3. Barraquer RI, Alvarez de Toledo JP, Alvarez Fischer M, Martínez Gran G. Prevención y tratamiento del astigmatismo en queratoplastia penetrante. Annals d'oftalmologia 2002; 10: 69-80.

4. Alpins N. A re-analysis of astigmatism correction (letter). Br J Ophthalmol 2002; 86: 832.

5. Shen Y, Tong J, Li YM. Corneal relaxing incision combined with phacoemulsification and IOL implantation. J Zhejiang Univ Sci 2004; 5: 985-988. 